\title{
Kinetics of Vapour Diffusion and Condensation in Natural Porous Cellulosic Fibre Web
}

\author{
Břetislav Češek, Miloslav Milichovský, and František Potůček \\ Department of Wood, Pulp, and Paper, Faculty of Chemical Technology, University of Pardubice, Studentská 95, 53210 Pardubice, \\ Czech Republic \\ Correspondence should be addressed to Břetislav Češek, bretislav.cesek@upce.cz
}

Received 2 June 2011; Accepted 7 July 2011

Academic Editors: E. Burkel and P. Sánchez

Copyright (๑) 2011 Břetislav Češek et al. This is an open access article distributed under the Creative Commons Attribution License, which permits unrestricted use, distribution, and reproduction in any medium, provided the original work is properly cited.

\begin{abstract}
Vapour diffusion in a porous web material followed by its absorption or condensation is a typical process for all porous materials particularly on polymeric and natural basis. The description of kinetic weight increment increase during this process was proposed by use of a relatively simple function with adequately defined theoretical background. It was shown that this kinetic process is controlled by vapour diffusion through porous web material. This tool in connection with observations at different air relative humidity and with use of relatively simple technique enables one to evaluate the structure properties and its structure homogeneity, that is, the uniformity character of the porous stratified web material. Utilization of this description was demonstrated during moistening kinetics of paper web of different composition from primary and secondary fibres on lignocellulosic and only cellulosic basis with different ash content.
\end{abstract}

\section{Introduction}

Condensation of liquid vapour in porous medium is an important phenomenon of behaviour of porous materials. Predominantly water is a key component controlling the behaviour of all biomaterials, living organisms, and so forth. We have distinguished the liquid water in porous systems as (i) self-organized water, that is, bulk of water whose behaviour is independent on the character and composition of porous system, and (ii) medium-enforced organized water, that is, water the organization of which in the porous system, particularly in microreticular system, is controlled by surface-molecular properties of components forming this system.

The states of liquid water are dependent on the specific surface area of pores. The medium-enforced organized water is typical for microporous and nanoporous media as natural so-called nanostructured polymer [1] being typically hygroscopic.

The hygroscopicity characterizes behaviour of porous materials and belongs to their very important properties. On the one hand, the hygroscopicity causes advantage of porous products; on the other hand, it causes some discrepancies of their quality. For instance, these discrepancies hold down application of paper products. Water-holding ability of paper material, foods, and other pore materials from surrounding air humidity is notorious. The complete understanding of moistening process is a necessary presumption for further paper expanse utilization, particularly for using of paper as packaging material $[2,3]$.

The amounts of received water and velocity of this process is dependent on a lot of factors. Except temperature, pressure, and relative humidity of air vicinity, the parameters as fibre components, degree of fibre beating, composition and amount of further fillers, degree of sizing, and other auxiliary paper agent's presence are important too. The porosity of paper material and its structure combined with the properties of pore interfaces appear to be the most important properties influencing hygroscopicity. Thus the hygroscopicity of porous material such as paper is dependent on both the porosity and the surface-molecular properties of porous material [4].

\section{Theoretical}

Generally, vapour absorption of water and of other liquids in porous materials is given by their microstructure and 
hypermolecular properties [4-6]. It was shown that the kinetics of moistening of paper and of the moulded fibre products $[4,6]$ and vapour absorption of organic liquids [7] is satisfactorly described by the following empirical equation:

$$
\Delta Y=\frac{a b+c t^{d}}{b+t^{d}}
$$

where $\Delta Y\left(=Y-Y_{i}\right)$ is the increment of moisture content (mass of liquid per unit mass of dry solid), given as the difference between current moisture content, $Y$, and initial moisture content at the beginning of the experiment, $Y_{i} . t$ is the time (in days) of sample storage at a given relative air humidity; $d$ characterizes the rate of steady-state adjustment; $c\left(=\Delta Y_{e}\right)$ is the hygroscopicity of the porous materials; $a$ and $b$ are empirical parameters.

The following two parameters, the steady-state moisture, $Y_{e}$, given as

$$
Y_{e}=\Delta Y_{e}+Y_{i}
$$

as well as the moistening rate at the beginning of the process, $v$, which is directly proportional to the parameters $b$ and $c$

$$
v \sim \frac{c}{1+b},
$$

indicate the relative steady-state moisture of the porous sample and the kinetics of moistening, respectively.

Parameter $v$ permits to evaluate the structure and hypermolecular properties of porous materials, for example, paper and paper products $[6,7]$. Equation (3) was obtained [6] by linearization of (1) at beginning of moistening or absorption process assuming that $a \rightarrow 0$.

Wet porosity, $\varepsilon_{r}$, that is, the steady-state volume capacity of all pores filled with condensed liquid at a given relative air humidity, can be expressed by the following equation $[5,6]$ :

$$
\varepsilon_{r}=\frac{\rho_{p} Y_{e}}{\rho_{l}},
$$

where $\rho_{l}$ is the density of condensed liquid $\left(\mathrm{kg} \mathrm{m}^{-3}\right) ; \rho_{p}$ is the apparent density of porous web material $\left(\mathrm{kg} \mathrm{m}^{-3}\right)$, for example, paper, given as the grammage of the porous sample divided by sample thickness.

The total porosity of the porous sample, $\varepsilon$, can be calculated on knowing the apparent density of the porous material $\rho_{p}$ :

$$
\varepsilon=1-\frac{\rho_{p}}{\rho_{s}},
$$

where $\rho_{s}$ is the density of the solid part of the porous material. In case of a multi-component material $\rho_{s}=1 /\left(\sum X_{i} / \rho_{i}\right)$, for $\sum X_{i}=1$, where $X_{i}$ and $\rho_{i}$ is absolute concentration (w/w) and density of the $i$ th component composed the solid part of the porous material, respectively. The cellulose has a density between 1540 and $1560 \mathrm{~kg} \mathrm{~m}^{-3}$ [6-8].

The interval of pores $\left(r_{\min } \leq r \leq r(\varphi)\right)$, expressed as the increment of porosity, $\Delta \varepsilon_{r}$, filled in steady-state with condensed liquid, at a given relative air humidity, $\varphi$, was defined as $[6,7]$ :

$$
\Delta \varepsilon_{r}=\varepsilon_{r}-\varepsilon_{r 0},
$$

where $\varepsilon_{r 0}$ is a porosity corresponds to the pores having the radius in the interval $0 \leq r \leq r_{\min }$, filled with condensed liquid at the beginning of condensation (given by (4) for $\left.Y_{e}=Y_{i}\right)$.

Both parameters $\Delta Y_{e}$ and $v$ are important but the parameter $v$ especially attracts attention as permitting to evaluate the structure and surface-molecular properties of the inner microwalls of the porous materials, for example, paper and fibre moulded products [7].

The process of vapour condensation, that is, vapour absorption, in porous web material takes place by diffusion of vapour from surrounding atmosphere into pores of porous medium following by quick condensation in suitable pores. The suitable pores are all pores which size characterized by equivalent cylindrical pore radius $r \leq r(\varphi)$. The value of maximal equivalent cylindrical pore radius, $r(\varphi)$, occupied by the condensed liquid, for example, water in the pore material at constant temperature and relative humidity, is expressed by the well-known Kelvin equation.

The kinetics of pore material moistening at the beginning of this process is characterized by the following assumptions at $t=0$ and under constant temperature and relative humidity [6]:

(i) initial condensed water fills only all the smallest pores with equivalent cylindrical pore radius $r \leq r_{\min }$;

(ii) water condensation in the porous material takes place predominantly through its surface pore layers;

(iii) vapour absorption, that is, condensation, takes place step by step and is controlled by diffusion of vapour from the surface layers into inner layers of the web porous material (Figure 1).

Given that the rate-limiting step of the absorption process is predominantly the one-dimensional diffusion of molecules in the perpendicular direction of the web porous material into its inner microthin layers (Figure 1) followed by their quickly condensation in suitable pores characterized by equivalent cylindrical pore radius $r \leq r(\varphi)$, this process is described by the well-known Fick's first law after simple rearrangement of this differential equation:

$$
-D \frac{\mathrm{d} C}{\mathrm{~d} t}=C_{o} \varphi\left(\frac{\mathrm{d} x}{\mathrm{~d} t}\right)^{2},
$$

where $C$ and $C_{o}$ are the actual vapour concentration and saturated vapour concentration of the measured component, respectively, at given condition.

If one can assume that

$$
Y=Y_{e} \frac{x}{x_{r a}},
$$

where $Y_{e}$ and $Y$ are the moisture contents of the condensed component at time $t \rightarrow \infty$ and time $t$ in both surface layers of width $2 x_{r a}$ and $2 x$, respectively. A full concentration 


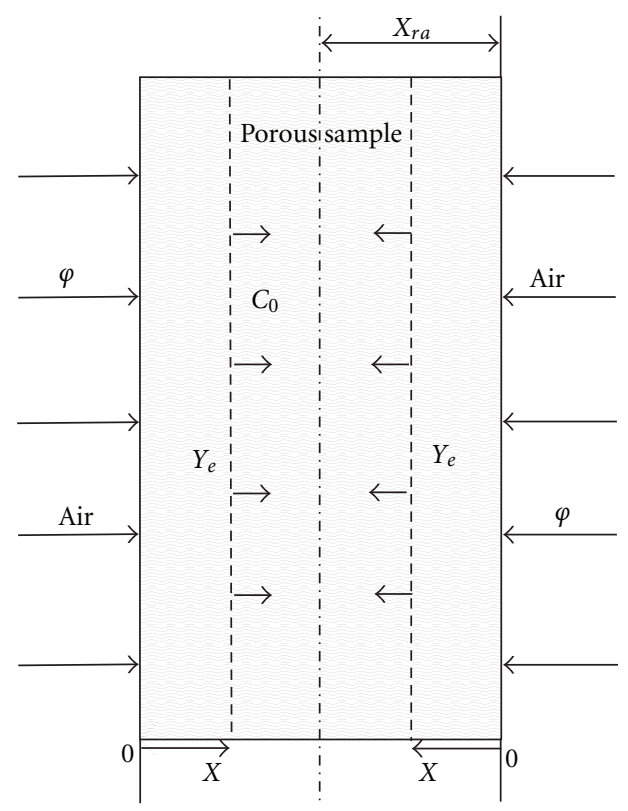

FIgURE 1: Schematic representations of one-dimensional twindirection vapour diffusion of the condensing liquid into porous web material.

change of the diffusing component followed by its absorption in the porous web, $d Y$, is in reality proportional to the relative concentration change of the condensed component in the layers of the porous web material, thus

$$
D \frac{\mathrm{d} Y}{\mathrm{~d} t}=\frac{C_{o} \varphi M \varepsilon}{\rho_{p}}\left(\frac{\mathrm{d} x}{\mathrm{~d} t}\right)^{2} .
$$

In comparison with analogical sorption processes taking place in the solution [9] which are characterized by the condition of a constant total concentration of the diffusing components realizable at any time of the sorption process, this process is typical with the constant concentration of the vapour of the diffusing component $\varphi C_{0}$.

If we assume that the boundary concentration gradient in porous web material is constant then we can define the velocity of this boundary penetration by the following function:

$$
\frac{\mathrm{d} x}{\mathrm{~d} t}=k^{0.5}\left(\frac{x}{t^{(d+1) / 2}}\right)
$$

where both variables are independent and $k$ is proportionality coefficient (optionally $k=1$ ). For this reason, the differential equation (9) can be connected with (8) and (10). Then, we can obtain a simple differential equation:

$$
D \frac{\mathrm{d} Y}{\mathrm{~d} t}=\frac{k C_{o} \varphi M \varepsilon Y^{2}}{\rho_{p}}\left(\frac{x_{r a}}{Y_{\mathrm{e}}}\right)^{2} \frac{1}{t^{d+1}}
$$

The parameter $d$ characterizes a uniformity of stratified structure of porous web material. If $d \rightarrow 1$, the porous web material has stratified structure with ideal uniformity, that is, all microlayers composed the stratified structure of the web porous material are the same. If $d>1$ or $d<1$, the porous web material has stratified structure with worse uniformity where vapour diffusion takes place slowly or rapidly than in an ideal web porous material, respectively, that is, the sample uniformity decreases.

By integrating (11) under the given boundary conditions from $Y=\Delta Y$ at $t=t$ to $Y=\Delta Y_{e}$ at $t \rightarrow \infty$, we can obtain the following relationship:

$$
Y=\frac{Y_{e} t^{d}}{\left(\varphi x_{r a}^{2} / D_{\mathrm{ps}} d Y_{e}\right)+t^{d}},
$$

where $D_{\mathrm{ps}}=D \rho_{p} / k C_{o} M \varepsilon$ or $D_{\mathrm{ps}}=D_{\mathrm{ps}}^{1} / d$, where $D_{\mathrm{ps}}^{1}=$ $\varphi x_{r a}^{2} / b Y_{e}$.

This description confirms that the diffusion of vapour into porous web form material as being the dominating process which controls the absorption kinetics, that is, the condensation of vapour in porous material, but in the dependence on its uniformity and structure homogeneity.

\section{Experimental Methods}

The experiments measured the kinetics of paper webs moistening and observed the influence of different primary pulp, secondary pulp (waste paper), and other fillers on this behaviour. Pulp defibrillation was carried out using a standard laboratory defibrillator according to ISO 5263-1. The total number of stirrer rotations was held at 30000 for all investigated pulps. To retain all the fibrous part of pulps in sheet, including short fibres and crill, the paper webs were prepared using Büchner funnel equipped with paper filter. After pulp slurry drainage, pressing and drying on laboratory hand-sheet machine followed, as usual. The grammage of all sheets was approximately the same. In all experiments, the pulps and groundwood were employed in sheet form, the groundwood after first drying only.

The kinetics of water vapour condensation in the paper samples was observed by using a gravimetrical method. All samples were acclimatized before the start of experiment for one week in the desiccator filled with dry silicagel at room temperature of $23 \pm 1^{\circ} \mathrm{C}$. At least three values measured were averaged to give a mean value reported in this paper.

A paper strip sample (approximately $2 \times 1 \mathrm{~cm}$ in size) was inserted in a glass weighing bottle of known weight. Sample's outer surface, $A$, varied between $4.0 \times 10^{-4}$ to $5.5 \times$ $10^{-4} \mathrm{~m}^{2}$, depending on thickness. The contact of the sample with the wall of the weighing bottle was minimal. The paper strip samples inside the weighing bottle were introduced in the desiccator filled with the prescribed solution of salts. The relative humidity in the desiccator was kept at three different levels, $97 \%, 75 \%$, and $49 \%$, which were achieved by means of saturated aqueous solutions of $\mathrm{K}_{2} \mathrm{SO}_{4}, \mathrm{NaCl}$, and $\mathrm{KNO}_{2}$, respectively. Thus this solution maintained a constant relative humidity in the desiccator without any air streaming. Before each balancing, the weighing bottle was air-proofed and, in a subsequent step, the weighing bottle was taken out of the desiccator and balanced.

Kinetic experiments were performed using primary and secondary fibres on cellulosic basis. The composition of 
TABLE 1: Characteristic of pulp fibres used in the experiments.

\begin{tabular}{|c|c|}
\hline Fibres & $\begin{array}{c}\text { Fibre } \\
\text { sample } \\
\text { number }\end{array}$ \\
\hline $\begin{array}{l}\text { Sulphate bleached pulp (mixture of Spruce and } \\
\text { Pine), Štětí, Czech Rep. }\end{array}$ & I \\
\hline $\begin{array}{l}\text { Sulphite bleached pulp MgBi (Spruce), Paskov, } \\
\text { Czech Rep. }\end{array}$ & II \\
\hline $\begin{array}{l}\text { Sulphate bleached pulp (NIST long fibre pulp } \\
\text { standard 8495) }\end{array}$ & III \\
\hline $\begin{array}{l}\text { Sulphate bleached hardwood pulp (Beech), } \\
\text { Ružomberok, Slovakia }\end{array}$ & IV \\
\hline $\begin{array}{l}\text { Sulphite nonbleached pulp CaBi (Spruce), Větřní, } \\
\text { Czech Rep. }\end{array}$ & $\mathrm{V}$ \\
\hline C12 waste paper, $46.6 \%$ of ash & VI \\
\hline C12 waste paper, $19 \%$ of ash & VII \\
\hline C12 waste paper, $4.7 \%$ of ash & VIII \\
\hline $\begin{array}{l}\text { Sulphate non-bleached pulp (mixture of Spruce and } \\
\text { Pine), Štětí, Czech Rep. }\end{array}$ & IX \\
\hline $\begin{array}{l}\text { Groundwood, pulp from Spruce taken from the } \\
\text { accepts stream of the primary screen, Czech Rep. }\end{array}$ & $\mathrm{X}$ \\
\hline $\begin{array}{l}\text { Sulphate hardwood bleached pulp from Eucalyptus } \\
\text { (NIST short fibre pulp standard 8496) }\end{array}$ & XI \\
\hline
\end{tabular}

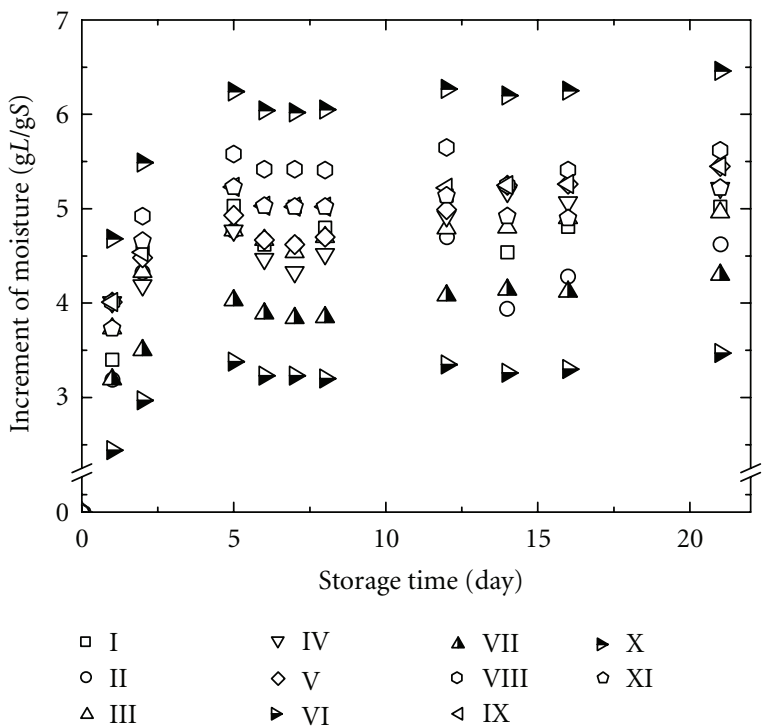

FIGURE 2: Kinetic of paper web moistening at atmosphere with $75 \%$ relative humidity (symbols: see Table 1.).

porous web materials tested on their hygroscopicity is described in Table 1. For testing of fibre moistening, bits of samples from their hand-sheets were used. All experiments were performed at standard room conditions (temperature of $23 \pm 1^{\circ} \mathrm{C}$ and relative humidity of $50 \pm 5 \%$ ).

\section{Data Treatment}

From experimental data, parameters $a, b, c$, and $d$ in (1) chosen to kinetic description were evaluated. Then, the moist- ening or condensation rate at the beginning of moistening or absorption process of the porous material, $v$, defined by (3), and steady-state moisture $Y_{e}$ of the paper samples with condensed liquids defined by (2) were also evaluated.

Under different air relative humidity values, that is,

(i)-low, R.H. $=49 \%$, condensed water filled the pores with a diameter lower than approximately $3 \mu \mathrm{m}$;

(j)-middle, R.H. $=75 \%$, condensed water filled the pores with a diameter lower than approximately $7 \mu \mathrm{m}$;

(k)-high, R.H. = 97\%, condensed water filled the pores with a diameter lower than approximately $70 \mu \mathrm{m}$. The decrement of relative void volume, $\Delta \varepsilon_{r}$, equivalent to the pores filled with liquid at steady state, can be calculated using (4) and (5), from which the following parameters could be also derived:

(i) $\left(\varepsilon-\varepsilon_{k}\right) / \varepsilon$-relative extent of big pores in the porous material, representing fraction of biggest pores filled with condensed water (pore diameter $>70 \mu \mathrm{m}$ at R.H. more than 97\%);

(ii) $\varepsilon_{i} / \varepsilon$-relative extent of the small pores in the porous material, representing fraction of small pores filled with condensed water (pore diameter $<3 \mu \mathrm{m}$ at R.H. equal to $49 \%$ );

(iii) $\left(\varepsilon_{k}-\varepsilon_{j}\right) / \varepsilon,\left(\varepsilon_{j}-\varepsilon_{i}\right) / \varepsilon$, and $\left(\varepsilon_{k}-\varepsilon_{i}\right) / \varepsilon$ - extent of the pore volume dispersion in the porous material $[4,7]$.

Under comparable conditions, that is, for the porous materials with approximately comparable mean pore sizes, the expressions $\left(\varepsilon_{k}-\varepsilon j\right) /\left(\varepsilon_{j}-\varepsilon_{i}\right)$, and $\left(\varepsilon_{k}-\varepsilon_{i}\right) / \varepsilon$ characterize an extent of the pore volume dispersion in the porous material providing relevant information on pore distribution function of porous system. Any increase in the values of these expressions renders the system more monodispersive, and vice versa. If value of so-called monodispersion factor $\left(\varepsilon_{k}-\right.$ $\left.\varepsilon_{i}\right) / \varepsilon \rightarrow 1$, the porous system is fully mono-dispersive.

By use of the Kelvin equation, it is possible to calculate the effective cylindrical pore radius of small pore $r_{i}$, middle pore $r_{j}$, and big pore $r_{k}$, as well as the contact angle, thus knowing hypermolecular properties of the porous material $[6,7]$.

\section{Results and Discussion}

An example of results of moistening experiments is depicted in Figure 2, for atmosphere with relative humidity equal to 75\% (symbols: see Table 1). The results of these experiments are summarized in Table 2. It is possible to claim that the function envisaged by (1) fits very well the experimental kinetic moistening data, with coefficient of determination approximately 0.99 (see Table 3 ). All experiments showed that at suitable experimental conditions the parameter $a$ of the kinetic equation (1) alternates around zero because the moisture of samples at start of moistening process was small (see Table 3).

The main hygroscopic parameters, the steady-state increment of sample moisture, $Y_{e}$, and the moistening rate at the start of moistening of pore sample, $v$, increases logically with increasing relative air humidity (Table 2). 
TABLE 2: Characteristic parameters of the paper webs moistening.

\begin{tabular}{|c|c|c|c|c|c|c|c|c|}
\hline $\begin{array}{l}\text { Fibre } \\
\text { sample no. }\end{array}$ & $\begin{array}{c}\rho_{p} \\
\left(\mathrm{~g} \mathrm{~cm}^{-3}\right) \\
\end{array}$ & $\begin{array}{c}x_{r a} \times 10^{3} \\
(\mathrm{~m})\end{array}$ & $d_{75}$ & $\begin{array}{c}v_{75} \\
(\operatorname{kg} L /(\operatorname{kg} S \text { day }))\end{array}$ & $\begin{array}{c}Y_{e}(75 \%) \\
(\operatorname{kg} L / \operatorname{kg} S)\end{array}$ & $d_{97}$ & $\begin{array}{c}v_{97} \\
(\operatorname{kg} L /(\operatorname{kg} S \text { day }))\end{array}$ & $\begin{array}{c}Y_{e}(97 \%) \\
(\operatorname{kg} L / \operatorname{kg} S)\end{array}$ \\
\hline I & 0.356 & 0.54 & 2.5503 & 3.40 & 0.1013 & 0.6999 & 6.88 & 0.2595 \\
\hline II & 0.320 & 0.59 & 3.3286 & 3.08 & 0.1022 & 0.7366 & 7.32 & 0.2374 \\
\hline III & 0.295 & 0.685 & 1.1161 & 3.73 & 0.1092 & 0.7066 & 7.19 & 0.2654 \\
\hline IV & 0.299 & 0.685 & 0.4936 & 3.91 & 0.1051 & 0.7688 & 6.93 & 0.2678 \\
\hline $\mathrm{V}$ & 0.321 & 0.6 & 0.6410 & 3.98 & 0.1105 & 0.6947 & 7.30 & 0.2657 \\
\hline VI & 0.396 & 0.525 & 1.5485 & 2.44 & 0.0739 & 0.7724 & 5.12 & 0.1778 \\
\hline VII & 0.362 & 0.59 & 0.4040 & 3.21 & 0.1029 & 0.6812 & 6.14 & 0.2158 \\
\hline VIII & 0.322 & 0.63 & 1.8553 & 4.01 & 0.1224 & 0.6992 & 8.15 & 0.2763 \\
\hline IX & 0.319 & 0.6 & 0.7419 & 4.03 & 0.1207 & 0.7370 & 7.55 & 0.2687 \\
\hline $\mathrm{X}$ & 0.203 & 0.945 & 1.1401 & 4.69 & 0.1421 & 0.7211 & 9.08 & 0.2903 \\
\hline XI & 0.336 & 0.6 & 2.2409 & 3.72 & 0.1117 & 0.6740 & 7.80 & 0.2936 \\
\hline
\end{tabular}

Subscripts 75 and 97 relate to relative humidity of $75 \%$ and of $97 \%$, respectively.

Table 3: Parameters in (1) for data depicted in Figure 2.

\begin{tabular}{|c|c|c|c|c|c|}
\hline Fibre sample no. & $\begin{array}{c}a \\
(\operatorname{kg} L / \operatorname{kg} S)\end{array}$ & $\begin{array}{c}b \\
\left(\text { day }^{d}\right)\end{array}$ & $\begin{array}{c}c \\
(\operatorname{kg} L / \operatorname{kg} S)\end{array}$ & $d$ & Coefficient of determination \\
\hline I & 0.00015 & 0.43659 & 4.88094 & 2.61364 & 0.985 \\
\hline II & 0.00029 & 0.42338 & 4.53498 & 3.47722 & 0.971 \\
\hline III & 0.00075 & 0.32199 & 5.00257 & 0.86804 & 0.996 \\
\hline IV & 0.00009 & 0.52118 & 5.95673 & 0.30326 & 0.979 \\
\hline V & 0.00010 & 0.48892 & 5.99125 & 0.35513 & 0.986 \\
\hline VI & 0.00011 & 0.31049 & 3.33876 & 1.96593 & 0.991 \\
\hline VII & 0.00083 & 0.40504 & 4.48784 & 0.48876 & 0.992 \\
\hline VIII & 0.00020 & 0.30266 & 5.45607 & 3.24407 & 0.989 \\
\hline IX & 0.00001 & 0.36628 & 5.49299 & 0.81650 & 0.996 \\
\hline $\mathrm{X}$ & 0.00001 & 0.35868 & 6.37877 & 1.11708 & 0.997 \\
\hline $\mathrm{XI}$ & 0.00040 & 0.36322 & 5.07241 & 2.24072 & 0.995 \\
\hline
\end{tabular}

Approximately, the paper samples No. X and XI, that is, the samples containing virgin lingocellulosic mechanical pulp and short-fibre primary chemical cellulosic pulp, have been moisturized more quickly and higher moisture content has been achieved. The highest moistening rate at all levels of the relative humidity was attained for groundwood, sample No. X. The secondary fibres (samples No. VI-VIII) reveal a strong dependence of both these parameters, the steady-state sample moisture, $Y_{e}$, and the moistening rate at the start of moistening, on the ash content of input resources (see Tables 1 and 2).

Porous structure of searched paper samples is assessed predominantly by the value of the term $\left(\varepsilon_{70}-\varepsilon_{7}\right) / \varepsilon$ (a subscript at $\varepsilon$ is pore diameter in $\mu \mathrm{m}$ ). With increasing the value of this term the polydispersion of pore size distribution decreases, and vice versa, this distribution is more monodispersive.

Relative minimal poly-dispersion of the porous structure appears for paper web composed of sulphate hardwood pulp No. XI followed by sample No. I composed of sulphate bleached softwood pulp. Surprisingly, the maximal polydispersion of the porous structure was achieved for sample
No. X composed of groundwood, because this fibre material is relatively formed predominantly by big pores. This fact is confirmed by the term $\left(\varepsilon-\varepsilon_{70}\right) / \varepsilon$ having the highest value for groundwood (0.904) from set of samples while the lowest value of $\varepsilon_{7} / \varepsilon(0.039)$ for this one corresponds to narrow extent of small pores.

Additionally, the characteristic results of the vapour diffusion and the web uniformity of stratified structure of searched porous web materials are summarized in Table 2. As results indicated, the vapour penetration is not dependent only on porous structure of paper web but a uniformity of this one across width of the web is also important. The web having high uniformity of stratified web structure is characterized by values of the uniformity parameter $d \rightarrow 1$, but this parameter is strongly influenced also by the amount of condensed water, that is, by the porous structure of web sample and air relative humidity. Comparing parameters $d_{75}$ and $d_{97}$ (a subscript at $d$ is relative humidity in \%) in Table 2, the uniformity of vapour-condensed water boundary movement across porous web is more uniform in air environment with high relative humidity. The vapour diffusion characterized by the pseudodiffusion coefficient, $D_{\mathrm{ps}}$, is 


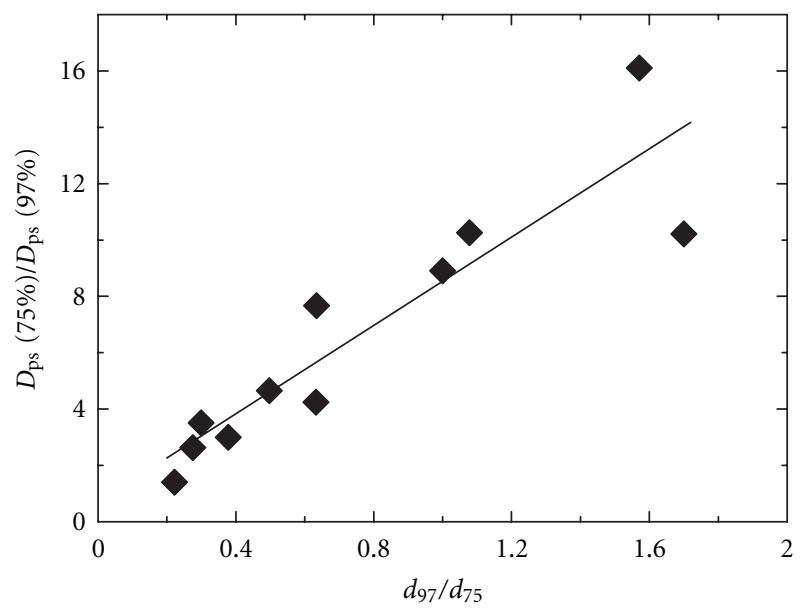

Figure 3: Dependence of the relative vapour pseudo diffusion coefficient of paper web on its relative uniformity.

strongly influenced by the amount of absorbed water in the porous web sample. With increasing the relative air humidity the pseudo diffusion coefficient significantly decreased because diffusion process is slowed down by strong condensation of water vapour in broad extent of free pores.

However, the pseudo diffusion coefficient is predominantly dependent on the uniformity of porous web during real diffusion process as documented in Figure 3. With relative decreasing value of the uniformity parameter the pseudo diffusion coefficient is increased. As it follows from Table 2, at relative humidity $75 \%$ the maximal vapour diffusion takes place relatively in samples No. VII and IV characterized with minimal values of $d_{75}$. On the other hand, the water vapour diffusion at high relative air humidity takes place slowly but the diffusion process is more uniform $\left(d_{97} \rightarrow 1\right)$.

At relative humidity $75 \%$, the values of $d_{75}$ alternate in high extent and in some cases are more than 1 . This fact indicates a high web nonuniformity of its stratified and two-sided structure appearing predominantly at low relative atmosphere humidity because the vapour have been condensed in small pores and the movement of the vapour-condensed water boundary through the wall of web sample is considerably blurred. Obviously, increasing the web two-sidedness, the value of parameter $d$ exceeds 1 but diffusion process is also dependent on a structure of porous web material.

From this reason, for better comparison of pseudo diffusion coefficient with the structure parameters of porous paper web it is reasonable to recalculate this one on standard condition, that is, for $d=1$. For this recalculation it is suitable to use (12) because $D_{\mathrm{ps}}^{1}=D_{\mathrm{ps}} d$. As documented in Figure 4, the standardized pseudo diffusion coefficient approximately linearly increases with increasing amount of free pores, un-filled with condensed water. Logically, water vapour diffusion through porous web material is improved if the relative amount of free pores without condensed water increases, that is, with a decrease of relative air humidity. Moreover, this process is deeply and more sensitively dominant in free porous structure of paper webs, that is, at low relative air humidity.

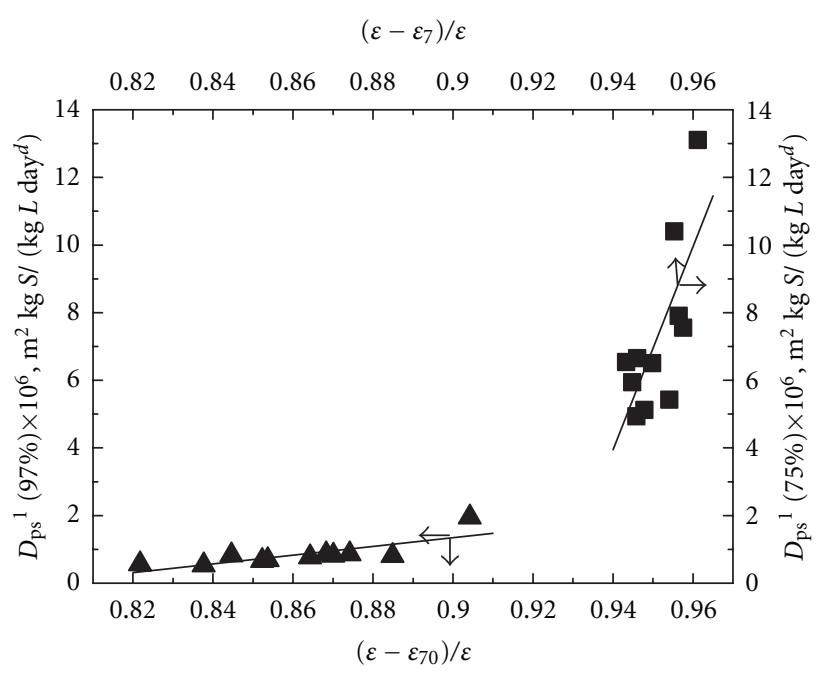

FIGURE 4: Dependence of standardized pseudo diffusion coefficient of paper web on relative amount of free pores un-filled with condensed water at relative humidity of $75 \%$ and of $97 \%$.

\section{Conclusion}

It was shown that the kinetics of important practical absorption vapour processes like vapour condensation and moistening of porous web material can be described using a relative simple function given by (1) with adequately defined theoretical background. Additionally, this tool enables in connection with moistening observations at different air relative humidity and by use of relatively simple technique to evaluate:

(i) the structure properties,

(ii) the structure homogeneity, that is, the uniformity character of the porous stratified web material.

Experimental conditions are limited only by strictly safe diffusion vapour process without any various movement of atmosphere in a glass weighing bottle cell.

In the case of paper web, it was observed that with increasing relative air humidity the water vapour diffusion is generally more regular but the diffusion process takes place slower because more and more amount of water vapour is condensed inside pore system and slowed down diffusion. All this process is influenced with both the ultrastructural features of pores and the uniformity character of porous web material. It is worth mentioning that this reality implies a utilization of the moistening rate at beginning of the process moistening or condensation as to be objective for further deeply description of structure and hypermolecular properties $[6,7]$ of the porous materials.

It was also confirmed that this proposed model is very well applicable to description of organic vapour absorption in porous web material [7]. This fact in connection with the results from moistening experiments allows to assess a deeply description of surface-molecular properties of pore walls in the porous web materials [7], that is, all the 
microstructural parameters and hypermolecular properties of these structures.

\section{Nomenclature}

A: $\quad$ Outer surface of the pore sample given by its geometry $\left(\mathrm{m}^{2}\right)$

a: $\quad$ Parameter in (1) $(\operatorname{kg} L / \operatorname{kg} S)$

$b: \quad\left(=x_{r a}^{2} \varphi /\left(d D_{\mathrm{ps}} Y_{e}\right)\right)$ parameter in $(1)\left(\right.$ day $\left.^{d}\right)$

$C, C_{0}$ : Vapour and saturated vapour concentration $\left(\mathrm{kmol} \mathrm{m}^{-3}\right)$

c: $\quad\left(=\Delta Y_{e}\right)$ parameter in (1) $(\mathrm{kg} L / \mathrm{kg} S)$

$D$ : Diffusion coefficient of the vapour of diffusing component through the porous web material $\left(\mathrm{m}^{2}\right.$ day $\left.^{-1}\right)$

$D_{\text {ps }}$ : Pseudo diffusion coefficient of the vapour of diffusing component through the porous web material $\left(\mathrm{m}^{2} \mathrm{~kg} S /\left(\mathrm{kg} L\right.\right.$ day $\left.\left.^{d}\right)\right)$

$D_{\mathrm{ps}}^{1}: \quad\left(=d D_{\mathrm{ps}}\right)$ standardized pseudo diffusion coefficient of the vapour of diffusing component through the porous web material recalculated on standardized uniformity of paper web, $(d=1)\left(\mathrm{m}^{2} \mathrm{~kg} S /\left(\mathrm{kg} L\right.\right.$ day $\left.\left.^{d}\right)\right)$

$d$ : $\quad$ Parameter characterizing a uniformity of stratified structure of porous web material (dimensionless)

$k$ : $\quad$ Proportionality coefficient $\left(\right.$ day $\left.^{(d-1)}\right)$

M: $\quad$ Molar mass $\left(\mathrm{kg} \mathrm{kmol}^{-1}\right)$

$r(\varphi)$ : Radius of equivalent cylindrical pore occupied by the condensed liquid, in our case water, in the pore material at $\varphi(\mathrm{m})$

$r_{\min }$ : Radius of maximal equivalent cylindrical pore being occupied by condensed water at beginning of humidification process $(\mathrm{m})$

$t$ : Time of sample storage at given relative humidity (day)

$v$ : Condensation or moistening rate at the beginning of vapour condensation or moistening of web pore sample $(\operatorname{kg} L /(\mathrm{kg} S$ day))

$x$ : Width of the surface layers of the porous web material filled with condensed liquid (m)

$x_{\text {ra }}:$ Half of sample thickness (m)

$Y$ : $\quad$ Current moisture content $(\operatorname{kg} L / \mathrm{kg} S)$

$Y_{\mathrm{e}}: \quad$ Steady-state moisture content, equilibrium value $(\operatorname{kg} L / \operatorname{kg} S)$

$Y_{\mathrm{i}}: \quad$ Initial moisture content $(\mathrm{kg} L / \mathrm{kg} S)$

$\Delta Y: \quad$ Increment of moisture content $(\operatorname{kg} L / \operatorname{kg} S)$

$\Delta Y_{\mathrm{e}}:$ Steady-state increment of moisture, equilibrium value $(\operatorname{kg~L} / \mathrm{kg} S)$.

\section{Greek Letters}

$\varepsilon:$ Total porosity of pore sample $(\mathrm{v} / \mathrm{v})$

$\varepsilon_{r}$ : Interval of pores $(0 \leq r \leq r(\varphi))$ filled in steady state by condensed water at a given $\varphi$ $(\mathrm{v} / \mathrm{v})$
$\Delta \varepsilon_{r}$ : Interval of pores $\left(r_{\min } \leq r \leq r(\varphi)\right)$ filled in steady state by condensed water at a given $\varphi(\mathrm{v} / \mathrm{v})$

$\rho_{l}:$ Density of condensed liquid $\left(\mathrm{kg} \mathrm{m}^{-3}\right)$

$\rho_{p}$ : Apparent density of paper mass, that is, grammage of paper sample divided by thickness of sample $\left(\mathrm{kg} \mathrm{m}^{-3}\right)$

$\rho_{\mathrm{s}}$ : Density of solid part of porous web material $\left(\mathrm{kg} \mathrm{m}^{-3}\right)$

$\varphi$ : Relative humidity of air.

\section{Abbreviations}

L: $\quad$ Liquid (in our case: water)

R.H.: Relative humidity

S: $\quad$ Solid (in our case: fibres dried on silicagel).

\section{Acknowledgments}

This paper was supported by the Ministry of Education, Youth, and Sports and Ministry of Culture of the Czech Republic under the Research Projects MSM0021627501 and NAKI DF11P01OVV028, respectively.

\section{References}

[1] M. Ioelovich, "Cellulose as a nanostructured polymer: a short review," BioResources, vol. 3, no. 4, pp. 1403-1418, 2008.

[2] M. Milichovský, "Water-the key phenomenon in production and utilisation of paper products," Chemicke Listy, vol. 94, no. 9, pp. 875-878, 2000.

[3] M. Milichovský, "Water as key substance in production and utilisation of paper products," Papir A Celuloza, vol. 55, no. 11, pp. 302-308, 2000.

[4] B. Češek and M. Milichovský, "Influence of porosity properties of paper products on theirs hygroscopicity behaviour," in Proceedings 15th International Papermaking Conference-Efficiency of Papermaking and Paper Converting Processes (PROGRESS '05), pp. 32/1-32/12, Wroclaw, Poland, September 2005.

[5] G. Adámková, M. Milichovský, and I. Nesládek, "Hygroscopicity of paper and paper products," Papir A Celuloza, vol. 57, no. 4, pp. 105-107, 2002.

[6] B. Cešek, M. Milichovský, and G. Adámková, "Evaluation of hygroscopicity kinetics of paper, moulded fibre products and other porous materials," Cellulose Chemistry and Technology, vol. 39, no. 3-4, pp. 277-302, 2005.

[7] B. Češek, M. Milichovský, and F. Potůček, "Evaluation of microstructural and hypermolecular properties of cellulosic materials in web form," Cellulose Chemistry and Technology, vol. 40, no. 9-10, pp. 705-717, 2006.

[8] S. Rolland du Roscoat, M. Decain, X. Thibault, C. Geindreau, and J. F. Bloch, "Estimation of microstructural properties from synchrotron X-ray microtomography and determination of the REV in paper materials," Acta Materialia, vol. 55, no. 8, pp. 2841-2850, 2007.

[9] M. Foglarova, J. Prokop, and M. Milichovský, "Oxidized cellulose: an application in the form of sorption filter materials," Journal of Applied Polymer Science, vol. 112, no. 2, pp. 669-678, 2009. 

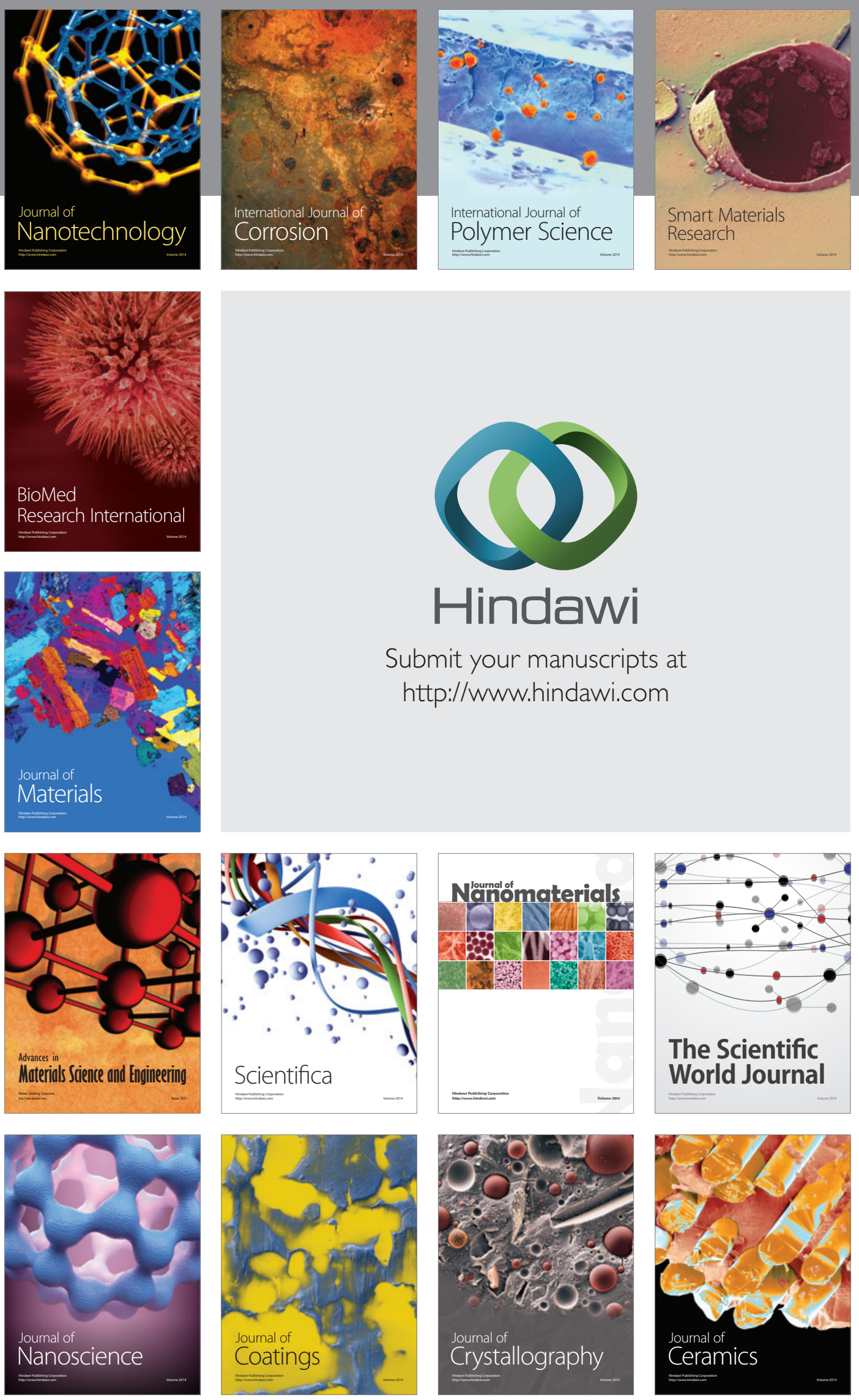

The Scientific World Journal

Submit your manuscripts at

http://www.hindawi.com

\section{World Journal}

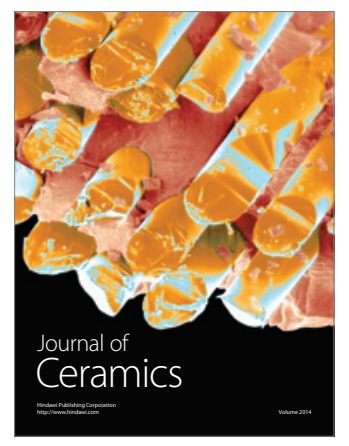

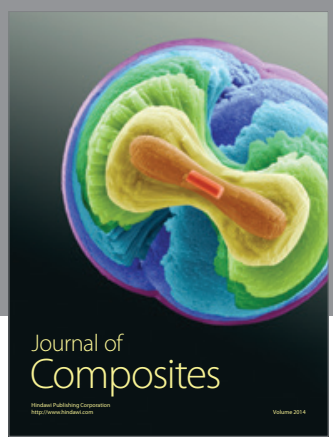
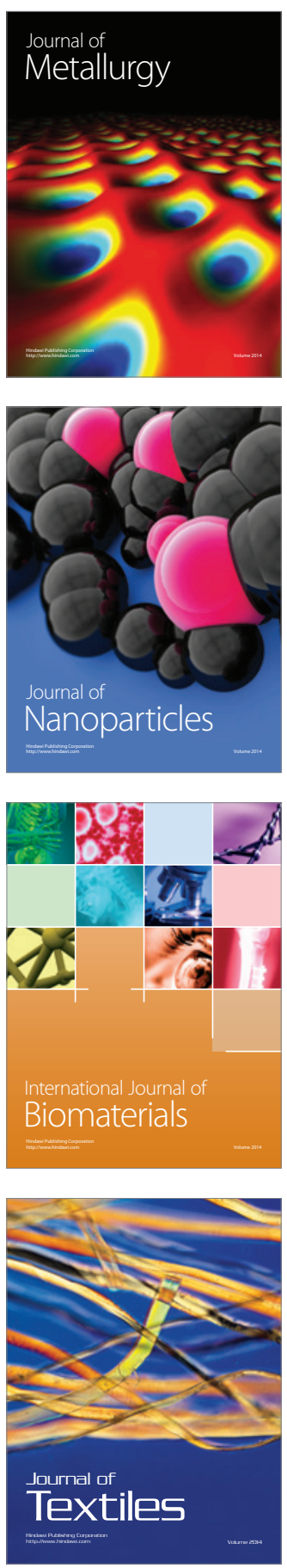